\title{
CONSTRUCTION ET CLASSIFICATION DE CERTAINES SOLUTIONS ALGÉBRIQUES DES SYSTĖMES DE GARNIER
}

\author{
par
}

Karamoko DIARRA

\begin{abstract}
Résumé. -
Dans cet article, nous classifions toutes les solutions algébriques (complètes) non élémentaires des systèmes de Garnier construites par la méthode de Kitaev : elles se déduisent des déformations isomonodromiques données en tirant en arrière une équation fuchsienne donnée $E$ par une famille de revêtements ramifiés $\Phi_{t}$. Nous introduisons tout d'abord les structures orbifoldes associées et sous-jacentes à une équation fuchsienne. Ceci nous permet d'avoir une version raffinée de la formule de Riemann Hurwitz qui nous permet rapidement de montrer que $E$ doit être hypergéométrique. Ensuite, on arrive à borner le degré de $\phi$ et les exposants, puis enfin à lister tous les cas possibles. Ceci généralise un résultat dû à $\mathrm{C}$. Doran dans le cas de l'équation de Painlevé VI. Nous construisons explicitement une de ces solutions.
\end{abstract}

\section{Introduction}

Rappelons qu'un système de Garnier de rang $N$ est un système d'équations différentielles d'ordre 2 non linéaire de rang $N$. D'après un théorème de Garnier [9], il est obtenu par la déformation isomonodromique de l'équation différentielle fuchsienne $E$ sur $\mathbb{P}^{1}$ avec $2 N+3$ pôles $(N+3$ points singuliers non apparents et $N$ singularités apparentes), qui s'écrit sous la forme normale $\frac{d^{2} u}{d x^{2}}+f(x) \frac{d u}{d x}+g(x) u=0$ avec

$$
\left\{\begin{array}{l}
f(x)=\frac{1-\theta_{0}}{x}+\frac{1-\theta_{1}}{x-1}+\sum_{i=1}^{N} \frac{1-\theta_{t_{i}}}{x-t_{i}}+\sum_{i=1}^{N} \frac{-1}{x-q_{i}} \\
g(x)=\frac{a}{x}+\frac{b}{x-1}-\sum_{i=1}^{N} \frac{H_{i}}{x-t_{i}}+\sum_{i=1}^{N} \frac{P_{i}}{x-q_{i}}
\end{array} .\right.
$$

Les solutions des systèmes de Garnier sont en général très transcendantes, mais ils peuvent avoir certaines solutions algébriques. Dans ce papier, on veut classer et construire explicitement certaines de ces solutions algébriques de Garnier. Si on commence par le cas le plus simple lorsque le rang $N=1$, le système de Garnier se réduit

Classification mathématique par sujets (2000). - 34M55, 34M56, 34M03.

Mots clefs. — Équations différentielles ordinaires, Déformations isomonodromiques, Familles de Hurwitz. 
à l'équation différentielle de Painlevé VI. On ne peut pas exprimer la solution générale de Painlevé VI à l'aide de solutions d'équations linéaires (ou même non linéaires du premier ordre) et d'opérations algébriques : Watanabe (dans [21]) montre que toute solution est ainsi transcendante excepté les solutions de type Riccati, et les solutions algébriques. Les premières apparaissent lorsque l'on considère les déformations isomonodromiques d'équations fuchsiennes réductibles (i.e. à monodromie triangulaire) : les coefficients de l'équation sont peu transcendants et satisfont une équation de Riccati. Watanabe 21 les a classifié, elles apparaissent pour des choix particuliers de valeurs propres. Les solutions algébriques apparaissent soit en familles, soit de manière sporadique et, si on en connaissait depuis Picard, la classification complète vient seulement d'être achevée par Lisovyy et Tykhyy [19]. Pour être plus exact, elle résulte de constructions de Picard, Hitchin ([10], 11]), Dubrovin, Mazzocco ([7], 8]), Kitaev ([16, [15, 17]) et surtout Boalch (44, [3]) qui a largement contribué à compléter la liste qui s'est ensuite avérée définitive : O. Lisovyy et Y. Tykhyy ont montré qu'il n'y en avait pas d'autres. On trouve (modulo symétries de Painlevé VI)

- 3 familles à un ou deux paramètres complexes,

- une famille à paramètre discret (points de torsion sur une courbe elliptique),

- 52 solutions sporadiques.

Bien que la motivation initiale de Painlevé était de trouver de nouvelles transcendantes, les solutions algébriques ont elles aussi un intérêt. Elles permettent chez Doran [6] de construire des fibrations elliptiques avec connexion de Gauss-Manin explicite, chez Boalch [5] de construire des solutions explicites au problème de Riemann-Hilbert, de construire des équations uniformisantes explicites pour certaines orbifoldes arithmétiques. D'après un travail de Dubrovin et Mazzocco [8], la transcendance de la solution générale des systèmes de Garnier augmente avec $N$ en ce sens qu'on ne peut intégrer le système de rang $N+1$ avec les solutions du système de rang $N$. Pour autant, il y aura encore des solutions spéciales, moins transcendantes qu'attendues, en considérant par exemple les déformations d'équations fuchsiennes réductibles, ou encore à monodromie finie. Les premières, étudiées par exemple dans le livre de Iwasaki, Kimura, Shimomura et Yoshida [13, se ramènent à des solutions d'équations différentielles linéaires. Les secondes vont produire des solutions algébriques du système de Garnier; c'est d'ailleurs comme cela que Boalch retrouve 49 des 52 solutions sporadiques de Painlevé VI. On pourrait tenter de classifier ce type de solutions algébriques, par exemple pour Garnier avec $N=2$, il y en aura beaucoup, mais ce sont plutôt les solutions algébriques correspondant à des déformations d'équations fuchsiennes non élémentaires (i.e. à monodromie Zariski dense) qui ont un intérêt. On les appellera "solutions algébriques non élémentaires". Dans le cas Painlevé VI $(N=1)$, l'équation d'isomonodromie a de nombreuses symétries, et partant d'une des 49 solutions algébrique élémentaires construites par Boalch, on peut déduire par symétrie une autre solution algébrique, qui sera bien souvent non élémentaire. Le but 
principal de ce papier est de construire des solutions algébriques non élémentaires des systèmes de Garnier pour $N>1$; aucune n'était connue avant. La méthode que nous allons utiliser est dûe à Kitaev [16] : elle permet de retrouver toutes les solutions algébriques de Painlevé VI modulo symétries. L'idée de départ est extrèmement simple; elle ne nécessite même pas de comprendre les équations d'isomonodromies. On fixe une équation fuchsienne disons $E$ sur $\mathbb{P}^{1}$, puis on la tire en arrière par une famille de revêtements ramifiés $\phi_{t}: \mathbb{P}^{1} \rightarrow \mathbb{P}^{1}$. On obtient alors une famille d'équations fuchsiennes $E_{t}:=\phi_{t}^{*} E$ qui, localement dans le paramètre $t$, là où la déformation est topologiquement triviale, est évidemment isomonodromique : la monodromie est essentiellement celle de l'équation $E$ en bas. Maintenant, si l'on prescrit le type topologique du revêtement, la famille $\phi_{t}$ forme une famille de Hurwitz : elle est algébrique et la déformation $E_{t}$ sera elle-même à coefficients algébriques. La difficulté principale pour mettre en pratique cette construction est que la dimension de déformation est en général trop petite face au nombre de pôles qu'aura $E_{t}$ : on ne construira qu'une solution algébrique partielle de l'équation d'isomonodromie, la solution complète restant en général transcendante. Si $E$ a $n$ pôles sur $\mathbb{P}^{1}$ et $\phi_{t}$ de degré $d$, alors $E_{t}$ aura en général $n d$ pôles; pour qu'elle soit élémentaire, on doit avoir $n \geq 3$ et l'espace de déformation de $E_{t}$ sera de dimension $N\left(E_{t}\right)=n d-3 \geq 3 d-3$. Pourtant, le nombre de paramètres libres dans la construction sera borné par le nombre de valeurs critiques de $\phi_{t}$, c'est-à-dire par $2 d-2$ d'après Riemann-Hurwitz : $2 d-2<<3 d-3=N\left(E_{t}\right.$ ) (on veut évidemment $d>1$ ). Pour obtenir une déformation algébrique complète, il faudra que $\phi_{t}$ ramifie suffisamment au dessus des pôles pour ne pas en avoir trop en haut; pour autant, elle doit ramifier suffisamment en dehors pour garder des paramètres libres dans la construction.

Considérons un exemple simple pour fixer les idées. Prenons pour $E$ une équation hypergéométrique (équation fuchsienne sur $\mathbb{P}^{1}$ à $n=3$ pôles simples en $z=0,1, \infty$ ) et considérons la famille de revêtements doubles $\phi_{t}: \mathbb{P}^{1} \rightarrow \mathbb{P}^{1}$ qui ramifie au dessus d'un des pôles, disons $z=\infty$, et d'un paramètre libre $z=t$. Alors l'équation fuchsienne $E_{t}=\phi_{t}^{*} E$ aura (pour $\left.t \neq 0,1, \infty\right) 5$ pôles, à savoir 2 au dessus de $z=0,2$ au dessus de $z=1$, et 1 au dessus de $z=\infty$; on n'aura qu'une solution partielle (de codimension 1) au système de Garnier correspondant (qui est ici de rang $N=2$ ). Par contre, si l'exposant de l'équation $E$ en $z=\infty$ est $\frac{1}{2}$ (i.e. la monodromie projective locale est d'ordre 2), alors la singularité de $E_{t}$ au dessus sera apparente (sans monodromie locale) et on pourra la chasser par une transformation de jauge méromorphe. On se retrouve alors avec une déformation à 1 paramètre d'équation fuchsienne $E_{t}^{\prime}$ à 4 pôles. Il est facile de vérifier que le birapport des 4 pôles varie avec $t$ : c'est une vraie déformation. C'est ainsi qu'on retrouve la famille de solutions algébriques de Painlevé VI à 2 paramètres (à savoir les exposants des pôles $z=0$ et $z=1$ ).

Il résulte d'un théorème de Klein [18 que toute équation fuchsienne à monodromie finie est, modulo transformation de jauge, le tiré-en-arrière $\phi^{*} E$ par un revêtement 
ramifié d'une équation hypergéométrique (plus précisément dans la liste de Schwarz [12], 20]). En particulier, toutes les solutions algébriques élémentaires construites par Boalch (et plus généralement leurs analogues pour les systèmes de Garnier) s'obtiennent aussi avec la méthode de Kitaev. Mais comme l'illustre l'exemple précédent, elle permet aussi de construire des solutions algébriques non élémentaires. Doran a classifié toutes celles que l'on pouvait construire de cette manière dans le cas de Painlevé VI : il retrouve les 3 familles (à un ou deux paramètres complexes) et 4 solutions sporadiques. Ces dernières s'obtiennent en tirant en arrière les hypergéométriques d'exposants $\left(\frac{1}{2}, \frac{1}{3}, \frac{1}{7}\right)$ et $\left(\frac{1}{2}, \frac{1}{3}, \frac{1}{8}\right)$ (groupes de triangles arithmétiques) par des revêtements de degré 10,12 et 18 . On montre que seuls les systèmes de Garnier de rang $N \leq 3$ ont des solutions complètes non élémentaires construites par la méthode de Kitaev. Elles sont listées dans les sections 3 et 5 . L'une d'elles est construite explicitement dans la dernière section. C'est une partie de ma thèse que j'ai fait sous la direction de F. Loray à l'université de Rennes 1.

\section{Structure orbifolde sous-jacente à une équation fuchsienne}

2.1. Structure orbifolde formelle. - Soit $X$ une surface de Riemann compacte. Une structure obifolde formelle sur $X$ est la donnée d'une famille finie $\left\{\left(t_{i}, p_{i}\right)\right\}_{i=1, \cdots, n}$ telle que :

$-t_{1}, \ldots, t_{n} \in X$ sont des points deux à deux distincts de $X$,

$-p_{1}, \ldots, p_{n} \in\{2,3,4,5, \ldots,+\infty\}$ sont des poids.

On peut encore la définir par une application

$$
p: X \rightarrow \mathbb{N}^{*} \cup\{\infty\}
$$

qui vaut 1 sauf pour un nombre fini de points, ici $\left\{t_{1}, \ldots, t_{n}\right\}$, son support.

On aura aussi à considérer des structures orbifoldes généralisées où cette fois

$$
p: X \rightarrow \mathbb{Q}_{+}^{*} \cup\{\infty\}
$$

(valant 1 sauf pour un nombre fini de points). Pour éviter toute confusion, on parlera de structure orbifolde entière concernant la première notion.

On définit la caractéristique d'Euler de la courbe orbifolde $\chi(X, p)$ par

$$
\chi(X, p):=2-2 g+\sum_{t \in X}\left(\frac{1}{p(t)}-1\right)=2-2 g-n+\frac{1}{p_{1}}+\cdots+\frac{1}{p_{n}}
$$

où $g=g(X)$ est le genre de la courbe $X$. Lorsque $p$ est à valeurs dans $\{1, \infty\}$, on retrouve la caractéristique d'Euler classique de la courbe épointée $X \backslash\{p=\infty\}$.

Si $\phi: Y \rightarrow X$ est un revêtement ramifié entre deux surfaces de Riemann compactes et si $p: X \rightarrow \mathbb{Q}_{+}^{*} \cup\{\infty\}$ est une structure orbifolde sur $X$, alors on définit le pull-back $\phi^{*} p$ par

$$
\phi^{*} p(t):=\frac{p(\phi(t))}{\operatorname{Ind}_{\phi}(t)} \quad \text { pour tout } t \in Y
$$


où $\operatorname{Ind}_{\phi}(t)$ est l'indice de $\phi$ en $t$, i.e. $\operatorname{Ind}_{\phi}(t)=k$ si $\phi(z)=z^{k}$ pour des coordonnées locales adéquates. Par exemple, si $p$ est la structure triviale $p \equiv 1$ sur $X$, alors $\phi^{*} p=\operatorname{Ind}_{\phi}$, la fonction indicielle.

\section{Proposition 2.1 (Riemann-Hurwitz version orbifolde)}

Si $\phi: Y \rightarrow X$ est un revêtement ramifié de degré $d$, alors $\chi\left(Y, \phi^{*} p\right)=d \cdot \chi(X, p)$.

Si $p$ et $p^{\prime}$ sont deux structures orbifoldes sur $X$, on dira que $p$ est inférieure à $p^{\prime}$ et on notera $p \leq p^{\prime}$ lorsque $p(t) \leq p^{\prime}(t)$ pour tout $t \in X$ (ici, $\infty=+\infty$ est un infiniment grand). On vérifie immédiatement que

$$
p \leq p^{\prime} \quad \Rightarrow \quad \chi(X, p) \geq \chi\left(X, p^{\prime}\right) .
$$

(Attention au renversement d'inégalités!)

On définit la structure orbifolde entière sous-jacente $\underline{p}$ à une structure orbifolde généralisée

$$
p: X \rightarrow \mathbb{Q}_{+}^{*} \cup\{\infty\}
$$

par

- si $p(t)=\frac{n}{q} \neq \infty$ alors $\underline{p}(t)=n$ où $(n, q)=1$;

- si $p(t)=\infty$ alors $\underline{p}(t)=\infty$.

Autrement dit, $\underline{p}(t)$ est le plus petit multiple entier de $p(t)$; en particulier, on a

$$
p(t) \leq \underline{p}(t) .
$$

Avant de voir le lien avec les équations fuchsiennes, rappelons le contexte géométrique dans lequel les structures orbifoldes apparaissent naturellement.

2.2. Structure orbifolde métrique et uniformisation. - Considérons un groupe fuchsien $\Gamma \subset \operatorname{PSL}(2, \mathbb{R})$ de type fini agissant proprement discontinument (par isométries) sur le demi-plan de Poincaré $\mathbb{H}$. Supposons en outre $\Gamma$ de co-volume fini, de sorte que $\mathbb{H} / \Gamma$ est une surface de Riemann compacte à laquelle on a enlevé un nombre fini de points. Notons $\phi: \mathbb{H} \rightarrow \mathbb{H} / \Gamma \subset X$. La fonction indicielle Ind $_{\phi}$ est constante sur les fibres de $\phi$ et on définit une structure orbifolde (entière) sur la courbe $X$ en posant

$$
\begin{aligned}
& -p(\phi(w)):=\operatorname{Ind}_{\phi}(w) \text { pour tout } w \in \mathbb{H}, \\
& -p(t)=\infty \text { si } t \in X \backslash(\mathbb{H} / \Gamma) .
\end{aligned}
$$

Le support de $p$ est précisément la réunion des valeurs critiques de $\phi$ et des pointes. Par construction, $\phi^{*} p$ (qui se définit localement comme dans la section précédente) est la structure orbifolde triviale sur $\mathbb{H}$.

La métrique de Poincaré $\mu$ sur $\mathbb{H}$ descend sur la courbe $X$ en une métrique singulière (à courbure constante -1 là où elle est lisse). Le support de la structure orbifolde $p$ est précisément la réunion des points singuliers de la métrique : l'angle de la surface 
$X$ autour d'un de ses points $t$ est donné par $\frac{2 \pi}{p(t)}$. Rappelons enfin la formule de Gauss-Bonnet dans ce cadre

$$
\operatorname{Aire}(X, \mu)=-2 \pi \chi(X, p) \text {. }
$$

On définit de la même manière la structure orbifolde d'un quotient de la sphère de Riemann $\mathbb{P}^{1}$ par un groupe fini d'isométries pour sa métrique à courbure constante +1 et on a (attention au signe)

$$
\operatorname{Aire}(X, \mu)=2 \pi \chi(X, p) .
$$

On peut enfin considérer les quotients du plan $\mathbb{C}$ par un groupe d'isométries euclidiennes, mais la caractéristique d'Euler $\chi(X, p)=0$ ne caractérise plus l'aire.

Théorème 2.2 (Klein-Poincaré). - Une surface de Riemann compacte munie d'une structure orbifolde entière $(X, p)$ est uniformisable, c'est à dire correspond à un des quotients décrits au dessus, si et seulement si on n'est pas dans l'une des situations suivantes :

- $\operatorname{genre}(X)=0$ et $\operatorname{support}(p)=\{t\}$ avec $p(t)<\infty$;

$-\operatorname{genre}(X)=0$ et $\operatorname{support}(p)=\left\{t_{1}, t_{2}\right\}$ avec $p\left(t_{1}\right) \neq p\left(t_{2}\right)$.

De plus, une orbifolde uniformisable est à courbure $>0$ (resp. $=0$ ou $<0)$ si et seulement si $\chi(X, p)>0($ resp. $=0$ ou $<0)$.

On en déduit la liste des orbifoldes uniformisables à courbure $>0$ :

$-\operatorname{genre}(X)=0$ et $\operatorname{support}(p)=\emptyset$,

- $\operatorname{genre}(X)=0$ et $\operatorname{support}(p)=\left\{t_{1}, t_{2}\right\}$ avec $p\left(t_{1}\right)=p\left(t_{2}\right)<\infty$,

- genre $(X)=0$ et $\operatorname{support}(p)=\left\{t_{1}, t_{2}, t_{3}\right\}$ où $p$ prend les valeurs :

$$
(2,2, k), k<\infty, \quad(2,3,3), \quad(2,3,4), \quad \text { ou } \quad(2,3,5) ;
$$

ainsi que celles de courbure nulle :

- $\operatorname{genre}(X)=0$ et $\operatorname{support}(p)=\{t\}$ avec $p(t)=\infty$,

- $\operatorname{genre}(X)=0$ et $\operatorname{support}(p)=\left\{t_{1}, t_{2}\right\}$ avec $p\left(t_{1}\right)=p\left(t_{2}\right)=\infty$,

- genre $(X)=0$ et $\operatorname{support}(p)=\left\{t_{1}, t_{2}, t_{3}\right\}$ où $p$ prend les valeurs

$$
(2,2, \infty), \quad(2,3,6), \quad(2,4,4), \quad \text { ou } \quad(3,3,3),
$$

- genre $(X)=0$ et $\operatorname{support}(p)=\left\{t_{1}, t_{2}, t_{3}, t_{4}\right\}$ où $p$ prend les valeurs $(2,2,2,2)$,

$-\operatorname{genre}(X)=1$ et $\operatorname{support}(p)=\emptyset$.

Rappelons que les aires $-2 \pi \chi$ d'orbifoldes (uniformisables) hyperboliques ne sont pas arbitrairement petites, mais bornées inférieurement par l'aire $\frac{\pi}{21}$ de l'orbifolde hypergéométrique $(2,3,7)$. Nous listons ci-dessous les orbifoldes d'aire $\leq \frac{\pi}{3}$ (c'est à dire pour lesquelles $-\chi \geq \frac{1}{6}$, on oublie systématiquement le facteur $2 \pi$ ); elles sont toutes hypergéométriques sauf une, de genre $g=0$ avec $n=4$ points orbifoldes. 


\begin{tabular}{|c|c|c|c|c|c|c|c|c|}
\hline$(2,3, p)$ & $(2,3,7)$ & $(2,3,8)$ & $(2,3,9)$ & $(2,3,10)$ & $(2,3,11)$ & $(2,3,12)$ & $\cdots$ & $(2,3, \infty)$ \\
\hline$-\chi=\frac{p-6}{6 p}$ & $\frac{1}{42}$ & $\frac{1}{24}$ & $\frac{1}{18}$ & $\frac{1}{15}$ & $\frac{5}{66}$ & $\frac{1}{12}$ & $\cdots$ & $\frac{1}{6}$ \\
\hline$(2,4, p)$ & $(2,4,5)$ & $(2,4,6)$ & $(2,4,7)$ & $(2,4,8)$ & $\cdots$ & $\cdots$ & $\cdots$ & $(2,4, \infty)$ \\
\hline$-\chi=\frac{p-4}{4 p}$ & $\frac{1}{20}$ & $\frac{1}{12}$ & $\frac{3}{28}$ & $\frac{1}{8}$ & $\cdots$ & $\cdots$ & $\cdots$ & $\frac{1}{4}$ \\
\hline$(2,5, p)$ & $(2,5,5)$ & $(2,5,6)$ & $(2,5,7)$ & $\cdots$ & $\cdots$ & $(2,5, \infty)$ & & $(2,6,6)$ \\
\hline$-\chi$ & $\frac{1}{10}$ & $\frac{2}{15}$ & $\frac{11}{70}$ & $\cdots$ & $\cdots$ & $\frac{3}{10}$ & & $\frac{1}{6}$ \\
\hline$(3,3, p)$ & $(3,3,4)$ & $(3,3,5)$ & $(3,3,6)$ & $\cdots$ & $(3,3, \infty)$ & & $(3,4,4)$ & $(2,2,2,3)$ \\
\hline$-\chi=\frac{p-3}{3 p}$ & $\frac{1}{12}$ & $\frac{2}{15}$ & $\frac{1}{6}$ & $\cdots$ & $\frac{1}{3}$ & & $\frac{1}{6}$ & $\frac{1}{6}$ \\
\hline
\end{tabular}

Enfin, $-\chi$ est minoré en fonction du genre $g$ et du nombre $n$ de points orbifoldes :

\begin{tabular}{|c|c|c|c|c|c|c|c|}
\hline$(g, n)$ & $(0,3)$ & $(0,4)$ & $(0,5)$ & $(0,6)$ & $(1,1)$ & $(1,2)$ & $(2,0)$ \\
\hline$-\chi \geq$ & $\frac{1}{42}$ & $\frac{1}{6}$ & $\frac{1}{2}$ & 1 & $\frac{1}{2}$ & 1 & 2 \\
\hline
\end{tabular}

2.3. Structure orbifolde et équations fuchsiennes. - Soit $E$ une équation fuchsienne sur $X$. On définit la structure orbifolde de $E$ (ou plutôt de la structure projective induite par $E$ sur $X$ ) de la manière suivante.

$-p(t)=1$ si $t \in X$ est un point régulier (i.e. non singulier) de l'équation $E$;

- $p(t)=\frac{1}{|\theta|} \in \mathbb{Q}^{+}$si $t \in X$ est un point singulier de l'équation $E$ d'exposant $\theta \in \mathbb{Q}$ non logarithmique (c'est-à-dire à monodromie périodique) ;

- $p(t)=\infty$ sinon $(t \in X$ est un point singulier de l'équation $E$ d'exposant $\theta \notin \mathbb{Q}$, ou encore un point singulier logarithmique d'exposant $\theta \in \mathbb{Z}$ ).

On notera $p(E)$ cette structure orbifolde. On a alors

Proposition 2.3. - Si E est une équation fuchsienne sur $X$ et $\phi: Y \rightarrow X$ un revêtement ramifié de degré $d$, alors

$$
p\left(\phi^{*} E\right)=\phi^{*} p(E) .
$$

La structure orbifolde sous-jacente $\underline{p}(E)$ ne dépend que de la monodromie de $E$ :

- si $t \in X$ est un point régulier (i.e. non singulier) de l'équation $E$, alors $\underline{p}(t)=1$;

- si $t \in X$ est un point singulier de l'équation $E$, alors $\underline{p}(t) \in\{2,3,4,5, \ldots,+\infty\}$ est l'ordre de la monodromie locale autour de $t$.

Nous observons que la structure orbifolde sous-jacente est invariante par transformations birationnelles sur l'équation. Par exemple, $\underline{p}(t)=1$ si $t$ est une singularité apparente.

Proposition 2.4. - Si E une équation fuchsienne sur $X$ et $\phi: Y \rightarrow X$ un revêtement ramifié de degré d, alors

$$
\underline{p}\left(\phi^{*} E\right) \geq \phi^{*} \underline{p}(E) .
$$


En particulier, $\chi\left(Y, \underline{p}\left(\phi^{*} E\right)\right) \leq d \cdot \chi(X, \underline{p}(E))$.

Si une orbifolde entière $(X, p)$ est uniformisable, i.e. définie par un revêtement ramifié $\phi: U \rightarrow X, U=\mathbb{P}^{1}, \mathbb{C}$ ou $\mathbb{H}$, alors la dérivée schwarzienne de $\phi^{-1}$ (qui ne dépend pas de la détermination choisie) définit une équation fuchsienne

$$
E: u^{\prime \prime}+\frac{S\left(\phi^{-1}\right)}{2} u=0
$$

sur $X$ dont la structure orbifolde est précisément $p$. Par exemple, on a

- si $X=\mathbb{P}^{1}$ et $\operatorname{support}(p)=\{0,1, \infty\}$, alors $E$ est l'équation hypergéométrique;

- si $X=\mathbb{P}^{1}$ et \#support $(p)=4$, alors $E$ est l'équation de Heun;

- si $X$ est une courbe elliptique et \#support $(p)=1$, alors $E$ est l'équation de Lamé.

Le résultat principal de cette section est la

Proposition 2.5. - Soit $E$ une équation fuchsienne sur $X$ dont la monodromie est non élémentaire, i.e. à image Zariski dense dans $\mathrm{PGL}(2, \mathbb{C})$. Alors sa structure orbifolde entière sous-jacente $(X, \underline{p})$ est une orbifolde uniformisable hyperbolique.

Démonstration. - Il suffit de montrer que si la structure orbifolde sous-jacente (qui est entière) n'est pas uniformisable ou n'est pas hyperbolique, alors l'équation fuchsienne $E$ est élémentaire. Rappelons que par transformation de jauge birationnelle, on peut ramener tous les exposants rationnels de $E$ dans l'intervalle $\left[0, \frac{1}{2}\right]$ sous l'action du groupe $\langle-\theta, \theta+1\rangle$, sauf peut-être un que l'on peut ramener dans l'intervalle $\left[\frac{1}{2}, 1\right]$ (sous le même groupe). Compte-tenu des listes au dessus, on doit donc en outre considérer les cas hypergéométriques $(g, n)=(0,3)$ avec exposants $\left(\frac{1}{2}, \frac{1}{2}, \frac{l}{k}\right),\left(\frac{1}{2}, \frac{1}{3}, \frac{2}{5}\right)$ et $\left(\frac{1}{3}, \frac{1}{3}, \frac{2}{3}\right)$. Mais tous ces triplets sont dans la liste de Schwarz des hypergéométriques à monodromie finie, donc élémentaires eux aussi.

2.4. Conséquences. - On cherche à classifier les couples $(E, \phi)$ où $E$ est une équation fuchsienne sur une surface de Riemann $X$ et $\phi: \tilde{X} \rightarrow X$ un revêtement ramifié (fini) tels que

- la monodromie de $E$ est non élémentaire,

- $\phi$ ramifie au dessus de $N \geq 3 \tilde{g}-3+\tilde{n}$ points distincts entre eux et distincts des singularités non apparentes de $E$

où $\tilde{g}$ est le genre de $\tilde{X}$ et $\tilde{n}$ le nombre de singularités non apparentes de $\tilde{E}$.

La dernière condition nous assure que $\phi$ peut se déformer avec $N$ paramètres indépendants, c'est à dire au moins la dimension de déformation isomonodromique de $\tilde{E}$ (dimension de l'espace de Teichmüller). On déduit de la section précédente les restrictions suivantes

Proposition 2.6. - Sous les hypothèses ci-dessus, on a $g(X)=g(\tilde{X})=0$ et on est dans l'un des cas suivants 
- E a 3 singularités non apparentes et $\operatorname{deg}(\phi) \leq 42$,

- E a 4 singularités non apparentes et $\operatorname{deg}(\phi) \leq 6$,

- E a 5 singularités non apparentes et $\operatorname{deg}(\phi) \leq 2$.

Démonstration. - On a (en supposant au pire des cas $\tilde{p}_{1}=\cdots=\tilde{p}_{\tilde{n}}=\infty$ pour la structure orbifolde de $\tilde{E})$

$$
-\chi\left(\phi^{*} E\right) \geq 2 \tilde{g}-2+\tilde{n}-N \geq 1-\tilde{g},
$$

et par ailleurs

$$
\chi\left(\phi^{*} E\right)=\operatorname{deg}(\phi) \cdot \chi(E) .
$$

Puisque l'on veut une déformation non élémentaire, on veut $\chi(E)<0$; donc $\tilde{g}=0$ (et donc $g=0$ aussi) et

$$
-\operatorname{deg}(\phi) \cdot \chi(E) \leq 1
$$

Évidemment, $\operatorname{deg}(\phi) \geq 2$ (sinon on n'a pas de déformation) et il vient

$$
-\chi(E) \leq \frac{1}{2}
$$

Maintenant, étant donné un couple $(E, \phi)$ satisfaisant aux 3 conditions précédentes, on considère la structure orbifolde sous-jacente, puis l'équation fuchsienne uniformisante correspondante $E^{\prime}$. Alors $\left(E^{\prime}, \phi\right)$ satisfait les même conditions que $(E, \phi)$. Par ailleurs, si $E^{\prime}$ satisfait les conclusions de la proposition, il en va de même de $(E, \phi)$. Donc on suppose dans la suite $E$ uniformisante. On conclut avec les estimations de la section précédente.

Les estimations obtenues sont encore très grossières. Examinons en détail les cas où $E$ a 4 ou 5 singularités non apparentes.

Proposition 2.7. - Sous les hypothèses précédentes, E a au plus 3 singularités.

Démonstration. - Comme dans la preuve de la précédente proposition, on suppose sans perte de généralité $E$ uniformisante et hyperbolique. Si $n=n(E)=4$, soit $p$ le maximum de sa structure orbifolde :

$$
-\chi(E) \geq\left(-2+\sum_{1}^{3}\left(1-\frac{1}{2}\right)+\left(1-\frac{1}{p}\right)\right)=\left(\frac{1}{2}-\frac{1}{p}\right) .
$$

Alors $p$ borne la structure orbifolde de $\tilde{E}=\phi^{*} E$ et on a

$$
-\chi(\tilde{E}) \leq\left(-2+\sum_{1}^{\tilde{n}}\left(1-\frac{1}{p}\right)-(\tilde{n}-3)\right)=1-\frac{\tilde{n}}{p} .
$$

Il vient

et donc

$$
d\left(\frac{1}{2}-\frac{1}{p}\right) \leq 1-\frac{\tilde{n}}{p}
$$

$$
d \leq 2 \frac{p-\tilde{n}}{p-2} .
$$


Bien sûr, on veut $\tilde{n}>3$ et $d>1$ pour obtenir une déformation non triviale; la seule possibilité reste donc $p=\infty$ et $d=2$. On vérifie aisément qu'il n'y a pas de déformation complète dans ce cas. Avec des arguments similaires, on exclut le cas $n=5$.

\section{Solutions algébriques non élémentaires pour le système de rang 2}

Ici, on suppose que $E$ est hypergéométrique, uniformisante hyperbolique, avec 3 pôles en $x=0,1, \infty$, que $\tilde{E}=\phi^{*} E$ a exactement 5 singularités non apparentes et que

$$
\phi: \mathbb{P}^{1} \rightarrow \mathbb{P}^{1}
$$

de degré $d=\operatorname{deg}(\phi)$ ramifie au dessus de 2 points distincts des pôles de $E$.

Proposition 3.1. - Sous les hypothèses précédentes, notons $\left(p_{0}, p_{1}, p_{\infty}\right)$ la structure orbifolde de $E$, avec $2 \leq p_{0} \leq p_{1} \leq p_{\infty} \leq \infty$ et $d:=\operatorname{deg}(\phi)$. Alors on a

$$
d\left(1-\frac{1}{p_{0}}-\frac{1}{p_{1}}-\frac{1}{p_{\infty}}\right) \leq 1-\frac{5}{p_{\infty}} .
$$

Démonstration. - Si on note $\left(\tilde{p}_{1}, \tilde{p}_{2}, \tilde{p}_{3}, \tilde{p}_{4}, \tilde{p}_{5}\right)$ la structure orbifolde sous-jacente de $\tilde{E}$, avec $2 \leq \tilde{p}_{1} \leq \tilde{p}_{2} \leq \tilde{p}_{3} \leq \tilde{p}_{4} \leq \tilde{p}_{5} \leq \infty$, alors on a $\tilde{p}_{5} \leq p_{\infty}$ : les singularités non apparentes en haut proviennent de celles d'en bas; leurs exposants sont des multiples de ceux d'en bas. On a ainsi

$$
-\chi(\tilde{E}) \leq 2 \tilde{g}-2+\sum_{i=1}^{5}\left(1-\frac{1}{\tilde{p_{i}}}\right)-2=1-\frac{1}{\tilde{p_{1}}}-\frac{1}{\tilde{p_{2}}}-\frac{1}{\tilde{p_{3}}}-\frac{1}{\tilde{p_{4}}}-\frac{1}{\tilde{p_{5}}} \leq 1-\frac{5}{p_{\infty}} .
$$

Par ailleurs

$$
d\left(1-\frac{1}{p_{0}}-\frac{1}{p_{1}}-\frac{1}{p_{\infty}}\right)=-d \cdot \chi(E) \leq-\chi(\tilde{E})
$$

d'où l'inégalité (11).

Proposition 3.2. - On est toujours sous les hypothèses précédentes.

1. On a l'égalité suivante :

$$
d-\left\lfloor\frac{d}{p_{0}}\right\rfloor-\left\lfloor\frac{d}{p_{1}}\right\rfloor-\left\lfloor\frac{d}{p_{\infty}}\right\rfloor=1
$$

où $\lfloor$ désigne la partie entière.

2. Si $p_{0} \leq d<p_{1} \leq p_{\infty}$ alors

$$
\frac{1}{p_{0}}+\frac{1}{d} \geq 1
$$

3. Si $p_{0} \leq p_{1} \leq d<p_{\infty}$ alors

$$
\frac{1}{p_{0}}+\frac{1}{p_{1}}+\frac{1}{d} \geq 1
$$


4. Si $p_{\infty} \leq d$ alors

$$
\frac{4}{5} \leq \frac{1}{p_{0}}+\frac{1}{p_{1}}<1
$$

Démonstration. - D'après Riemann-Hurwitz, $\phi$ possède $2 d-2$ points de ramification comptés avec multiplicité dont 2 au moins s'envoient en dehors de $x=0,1, \infty$. Le nombre de points au dessus de $x=0,1, \infty$ est donc minoré par

$$
\# \phi^{-1}(\{0,1, \infty\}) \geq 3 d-(2 d-2)+2=d+4 .
$$

Le nombre de singularités apparentes de $\tilde{E}$ au dessus de $x=i$ est majoré par $\left\lfloor\frac{d}{p_{i}}\right\rfloor$, $i=0,1, \infty$. Ainsi, le nombre de singularités non apparentes de $\tilde{E}$ (c'est à dire 5 ) est minoré par

$$
5 \geq d+4-\left\lfloor\frac{d}{p_{0}}\right\rfloor-\left\lfloor\frac{d}{p_{1}}\right\rfloor-\left\lfloor\frac{d}{p_{\infty}}\right\rfloor .
$$

D'un autre côté, $\left\lfloor\frac{d}{p_{i}}\right\rfloor \leq \frac{d}{p_{i}}$ et on a

$d+4-\left\lfloor\frac{d}{p_{0}}\right\rfloor-\left\lfloor\frac{d}{p_{1}}\right\rfloor-\left\lfloor\frac{d}{p_{\infty}}\right\rfloor \geq d+4-\frac{d}{p_{0}}-\frac{d}{p_{1}}-\frac{d}{p_{\infty}} \geq 4+d\left(1-\frac{1}{p_{0}}-\frac{1}{p_{1}}-\frac{1}{p_{\infty}}\right)>4$

( $E$ est hyperbolique) et puisque le terme de gauche est entier, on obtient

$$
d+4-\left\lfloor\frac{d}{p_{0}}\right\rfloor-\left\lfloor\frac{d}{p_{1}}\right\rfloor-\left\lfloor\frac{d}{p_{\infty}}\right\rfloor \geq 5
$$

d'où l'égalité (2). Si $p_{\infty}>d$, on a $\left\lfloor\frac{d}{p_{\infty}}\right\rfloor=0$ et l'équation (2) devient $d-\left\lfloor\frac{d}{p_{0}}\right\rfloor-\left\lfloor\frac{d}{p_{1}}\right\rfloor=$ 1. On sait que $d\left(1-\frac{1}{p_{0}}-\frac{1}{p_{1}}\right) \leq d-\left\lfloor\frac{d}{p_{0}}\right\rfloor-\left\lfloor\frac{d}{p_{1}}\right\rfloor=1$ alors on a $\frac{1}{p_{0}}+\frac{1}{p_{1}}+\frac{1}{d} \geq 1$.

Si $p_{\infty} \leq d$, d'après l'hyperbolicité de $E$, on a $\frac{1}{p_{0}}+\frac{1}{p_{1}}<1$. On remplace $d$ par $p_{\infty}$ dans l'inégalité (11) et on obtient l'inégalité $\left(1-\frac{1}{p_{0}}-\frac{1}{p_{1}}\right) p_{\infty}^{2}-2 p_{\infty}+5 \leq 0$. Pour qu'elle admette une solution, son discriminant doit être supérieur ou égal à 0 . Ainsi on a $\frac{1}{p_{0}}+\frac{1}{p_{1}} \geq \frac{4}{5}$.

Remarque 3.3. - De la preuve de l'égalité (2), on déduit que $\phi$ réalise nécessairement le nombre maximum possible de points singuliers apparents pour $\tilde{E}$ au dessus de $x=0,1, \infty$. Autrement dit, au dessus de $x=0$ par exemple, $\phi$ a exactement $\left\lfloor\frac{d}{p_{0}}\right\rfloor$ points ramifiant à l'ordre $p_{0}-1$ (d'indice $p_{0}$ ).

Examinons d'abord le cas $d<p_{\infty}$. Les inégalités (3) et (41) nous donnent les possibilités suivantes :

\begin{tabular}{|c|c|}
\hline Triplets $\left(p_{0}, p_{1}, p_{\infty}\right)$ & Degrés de revêtement $d$ \\
\hline$\left(2, p_{1}, p_{\infty}\right)$ & 2 \\
\hline$\left(2,3, p_{\infty}\right)$ & $3,4,5,6$ \\
\hline$\left(2,4, p_{\infty}\right)$ & 4 \\
\hline$\left(3,3, p_{\infty}\right)$ & 3 \\
\hline
\end{tabular}


Les paramètres $p_{1}$ et $p_{\infty}$ du tableau sont des entiers naturels suffisamment grands pour vérifier la condition d'hyperbolicité, par hypothèse $>d$, ou infinis. Puisque ces paramètres n'interviennent pas dans les contraintes de la construction, on peut tout aussi bien les fixer à $\infty$, ce que nous ferons ensuite. Dans chacun des cas, on vérifie si la condition (2) est satisfaite; c'est toujours le cas sauf pour le revêtement de degré 5. En tenant compte du fait que $\phi$ doit avoir le nombre maximal de points singuliers apparents (voir remarque précédente), on trouve la liste suivante

\begin{tabular}{|c|c|c|c|}
\hline Triplets $\left(p_{0}, p_{1}, p_{\infty}\right)$ & Degrés $d$ & Type de ramifications & $N$ \\
\hline$(2, \infty, \infty)$ & 2 & $(2 ; 1+1 ; 1+1)$ & 1 \\
\hline & 3 & $(2+1 ; 3 ; 1+1+1)$ & 1 \\
$(2,3, \infty)$ & 4 & $(2+2 ; 3+1 ; 1+1+1+1)$ & 2 \\
& 6 & $(2+2+2 ; 3+3 ; 1+1+1+1+1+1)$ & 3 \\
\hline$(2,4, \infty)$ & 4 & $(2+2 ; 4 ; 1+1+1+1)$ & 1 \\
\hline$(3,3, \infty)$ & 3 & $(3 ; 3 ; 1+1+1)$ & 0 \\
\hline
\end{tabular}

Par exemple, pour la ligne 3, les ramifications au dessus de $x=0,1$ sont imposées et il nous reste 2 points de ramification que l'on peut choisir fixe au dessus de $x=$ $\infty$ ou libre; dans chacun des cas, la condition (2) nous assure que la déformation est complète. On a systématiquement privilégié de maximiser le nombre de points libres dans le tableau, les autres cas de figure s'en déduisent facilement. Par exemple, toujours pour la ligne 3 , on a le cas dégénéré de type Painlevé VI $(2+2 ; 3+1 ; 2+1+1)$. Seules les lignes 3 et 4 nous fournissent des solutions possibles pour les systèmes de Garnier avec $N>1$. Examinons maintenant le cas $p_{0} \leq p_{1} \leq p_{\infty} \leq d$. L'inégalité (5) nous dit que $\left(p_{0}, p_{1}\right)=(2,3)$ puis en utilisant (1) et le fait que $p_{\infty} \leq d$, on trouve la liste suivante

\begin{tabular}{|c|c|}
\hline Triplets $\left(p_{0}, p_{1}, p_{\infty}\right)$ & Degrés de revêtement $d$ \\
\hline$(2,3,7)$ & $7,8,9,10,11,12$ \\
\hline$(2,3,8)$ & 8,9 \\
\hline
\end{tabular}

Seul le revêtement de degré 11 ne satisfait pas l'égalité (2). Pour les autres, on trouve 


\begin{tabular}{|c|c|c|c|}
\hline Triplets $\left(p_{0}, p_{1}, p_{\infty}\right)$ & Degrés $d$ & Type de ramifications & $N$ \\
\hline & 7 & $(2+2+2+1 ; 3+3+1 ; 7)$ & -1 \\
& 8 & $(2+2+2+2 ; 3+3+1+1 ; 7+1)$ & 0 \\
$(2,3,7)$ & 9 & $(2+2+2+2+1 ; 3+3+3 ; 7+1+1)$ & 0 \\
& 10 & $(2+2+2+2+2 ; 3+3+3+1 ; 7+1+1+1)$ & 1 \\
& 12 & $(\underbrace{2+\cdots+2}_{6 \text { fois }} ; 3+3+3+3 ; 7+\underbrace{1+\cdots+1}_{5 \text { fois }})$ & 2 \\
& & $(2+2+2+2 ; 3+3+1+1 ; 8)$ & -1 \\
\hline$(2,3,8)$ & 8 & $(2+2+2+2+1 ; 3+3+3 ; 8+1)$ & -1 \\
\hline
\end{tabular}

Les lignes avec $N=-1$ correspondent à $\tilde{n}=2$ singularités en haut : il n'y a certainement pas de revêtement correspondant. Les lignes $N=0$, lorsque le revêtement existe, vont nous donner de nouveau une équation hypergéométrique en haut. La ligne $N=1$ nous donne une des solutions algébriques de Painlevé VI dans la liste de Doran. La seule possibilité pour nous est la ligne 5 avec $N=2$. On a démontré le

Théorème 3.4. - Si E est une équation hypergéométrique non élémentaire dont les monodromies locales sont d'ordre $\left(p_{0}, p_{1}, p_{\infty}\right)$ et si $\phi: \mathbb{P}^{1} \rightarrow \mathbb{P}^{1}$ est une famille à 2 paramètres de revêtements ramifiés tel que $\phi_{t}^{*} E$ a 5 singularités non apparentes, alors on est dans la liste:

\begin{tabular}{|c|c|c|}
\hline Triplets $\left(p_{0}, p_{1}, p_{\infty}\right)$ & Degrés $d$ & Type de ramifications \\
\hline$(2,3, \infty)$ & 4 & $(2+2 ; 3+1 ; 1+1+1+1)$ \\
\hline$(2,3, \infty)$ & 6 & $(2+2+2 ; 3+3 ; 2+1+1+1+1)$ \\
\hline$(2,3,7)$ & 12 & $(\underbrace{2+\cdots+2}_{6 \text { fois }} ; 3+3+3+3 ; 7+1+1+1+1+1)$ \\
\hline
\end{tabular}

TABLE 1. Listes Pull-back hypergéométriques

Dans le tableau, $\left(p_{0}, p_{1}, p_{\infty}\right)$ désigne l'ordre local des monodromies de l'hypergéométrique $E$ aux point singuliers 0,1 et $\infty$, la seconde colonne donne le degré de $\phi_{t}$ et la colonne de droite, la partition des fibres de $\phi_{t}$ au dessus de 0,1 et $\infty$.

Remarque 3.5. - Dans ce théorème, on constate qu'il n'existe pas de pull-back à 2 paramètres libres de triangles hyperboliques $(2, \infty, \infty),(2,3,8),\left(2,4, p_{\infty}\right)$ et $\left(3,3, p_{\infty}\right)$ où $p_{\infty}$ est un entier naturel très grand. Les triangles hyperboliques $(2,3, \infty)$ et $(2,3,8)$ admettent des pull-back à 1 paramètre de degrés 3 et 9 respectivement; on a

Pour $d=3:(2,3, \infty) ;(2+1 ; 1+1+1 ; 3)$

Pour $d=9$ : $(2,3,8) ;(2+2+2+2+1 ; 3+3+1+1+1 ; 8+1)$.

Ceci construit des solutions algébriques incomplètes. 
3.1. Existence des revêtements. - Le tableau des ramifications 1 satisfait la formule de Riemann-Hurwitz, ce qui ne suffit pas à montrer l'existence des revêtements ramifiés correspondant. Dans la dernière section, on donnera des expressions explicites pour le revêtement concernant la lignes 1 du tableau. Pour la 3ième ligne, les calculs sont trop compliqués; on démontre l'existence comme suit.

Il faut construire le revêtement topologique $\phi: \mathbb{S}^{2} \rightarrow \mathbb{P}^{1}:$ il existera alors une unique structure complexe sur la sphère $\mathbb{S}^{2}$ rendant la flêche holomorphe, et donc rationnelle. L'existence d'un revêtement topologique se démontre en construisant une représentation

$$
\pi_{1}\left(\mathbb{P}^{1} \backslash\{\text { valeurs critiques }\}\right) \rightarrow \operatorname{Perm}\{1, \ldots, d\}
$$

où $d$ est le degré du revêtement : on le construit via sa monodromie. En fait, le tableau de ramification complète est

$$
(\underbrace{2+\cdots+2}_{6 \text { fois }} ; 3+3+3+3 ; 7+1+1+1+1+1 ; \underbrace{1+\cdots+1}_{10 \text { fois }}+2 ; \underbrace{1+\cdots+1}_{10 \text { fois }}+2)
$$

au dessus des points critiques

$$
\left(0,1, \infty, \lambda_{1}, \lambda_{2}\right)
$$

Il faut donc trouver des permutations $\sigma_{0}, \sigma_{1}, \sigma_{\infty}, \tau_{1}, \tau_{2} \in \operatorname{Perm}\{1, \ldots, d\}$ conjuguées individuellement à

$$
\begin{gathered}
\sigma_{0} \sim(12)(34)(56)(78)(910)(1112), \quad \sigma_{1} \sim(123)(456)(789)(101112), \quad \sigma_{\infty} \sim(1234567) \\
\tau_{1} \sim \tau_{2} \sim(12) \quad \text { avec } \sigma_{0} \circ \sigma_{1} \circ \sigma_{\infty} \circ \tau_{1} \circ \tau_{2}=\text { identité. }
\end{gathered}
$$

En fait, on trouve chez Doran et Kitaev une famille à 1 paramètre de revêtements ramifiés du type

$$
(\underbrace{2+\cdots+2}_{6 \text { fois }} ; 3+3+3+3 ; 7+2+1+1+1 ; \underbrace{1+\cdots+1}_{10 \text { fois }}+2)
$$

C'est une sous-famille de celle que nous cherchons correspondant à une confluence : une des deux ramifications libres, disons $\lambda_{1}$, est à l'infini. Correspond alors un revêtement topologique et une représentation donnée par

$$
\begin{gathered}
\sigma_{0}^{\prime} \sim(12)(34)(56)(78)(910)(1112), \quad \sigma_{1}^{\prime} \sim(123)(456)(789)(101112), \quad \sigma_{\infty}^{\prime} \sim(1234567)(89) \\
\tau_{2}^{\prime} \sim(12) \quad \text { avec } \sigma_{0}^{\prime} \circ \sigma_{1}^{\prime} \circ \sigma_{\infty}^{\prime} \circ \tau_{2}^{\prime}=\text { identité. }
\end{gathered}
$$

Puisque $(1234567)(89)=(1234567) \circ(89)$, il est clair que l'on peut décomposer $\sigma_{\infty}^{\prime}=$ $\sigma_{\infty} \circ \tau_{1}$, ce qui montre l'existence du revêtement. 
3.2. Exposants $\left(\tilde{\theta}_{0}, \tilde{\theta}_{1}, \tilde{\theta}_{t_{1}}, \tilde{\theta}_{t_{2}}, \tilde{\theta}_{\infty}\right)$. - On a travaillé jusque maintenant avec la structure orbifolde sous-jacente à l'équation $E$. Pour chaque type de revêtement listé dans le théorème 3.4 on peut choisir pour $E$ toute équation dont la structure orbifolde sous-jacente est donnée par $\left(p_{0}, p_{1}, p_{\infty}\right)$.

Par exemple, si on considère la première ligne du tableau 1] en prenant pour $E$ l'équation hypergéométrique d'exposants $\left(\frac{1}{2}, \frac{1}{3}, \theta_{\infty}\right), \theta_{\infty} \in \mathbb{C}$ arbitraire, on aura après pull-back une famille isomonodromique d'équations fuchsiennes avec 5 pôles non apparents d'exposants

$$
\left(\frac{1}{3}, \theta_{\infty}, \theta_{\infty}, \theta_{\infty}, \theta_{\infty}\right)
$$

et deux pôles apparents d'exposant 2. Si, par contre, on part de l'équation hypergéométrique d'exposants $\left(\frac{1}{2}, \frac{2}{3}, \theta_{\infty}\right)$, on obtiendra alors une famille d'équations fuchsiennes avec 5 pôles non apparents d'exposants

$$
\left(\frac{2}{3}, \theta_{\infty}, \theta_{\infty}, \theta_{\infty}, \theta_{\infty}\right)
$$

et trois pôles apparents d'exposant 2. Pourtant, les deux familles ainsi obtenues sont reliées par une équivalence de jauge birationnelle. En fait, on peut voir cette équivalence en bas, sur les hypergéométriques :

$$
\left(\frac{1}{2}, \frac{2}{3}, \theta_{\infty}\right)=\left(1-\frac{1}{2}, 1-\frac{1}{3}, \theta_{\infty}\right) \sim\left(\frac{1}{2}, \frac{1}{3}, \theta_{\infty}\right) .
$$

Cette équivalence de jauge se relève en une équivalence de jauge entre les deux familles isomonodromiques. Il nous faut donc classer les équations fuchsiennes pour chacune des 3 orbifoldes uniformisantes donnée par le tableau 1 modulo équivalence de jauge birationnelle. On trouve le tableau suivant :

\begin{tabular}{|c|c|c|}
\hline Degrés & Exposants de $E$ & Exposants de $E^{\prime}$ \\
\hline 3 & $\left(\frac{1}{2}, \frac{1}{3}, \theta_{\infty}\right)$ & $\left(\frac{1}{2}, \frac{1}{3}, \frac{1}{3}, \frac{1}{3}, 3 \theta_{\infty}\right)$ \\
\hline 4 & $\left(\frac{1}{2}, \frac{1}{3}, \theta_{\infty}\right)$ & $\left(\frac{1}{3}, \theta_{\infty}, \theta_{\infty}, \theta_{\infty}, \theta_{\infty}\right)$ \\
\hline 6 & $\left(\frac{1}{2}, \frac{1}{3}, \theta_{\infty}\right)$ & $\left(2 \theta_{\infty}, \theta_{\infty}, \theta_{\infty}, \theta_{\infty}, \theta_{\infty}\right)$ \\
\hline 12 & $\left(\frac{1}{2}, \frac{1}{3}, \frac{1}{7}\right)$ & $\left(\frac{1}{7}, \frac{1}{7}, \frac{1}{7}, \frac{1}{7}, \frac{1}{7}\right)$ \\
& $\left(\frac{1}{2}, \frac{1}{3}, \frac{2}{7}\right)$ & $\left(\frac{2}{7}, \frac{2}{7}, \frac{2}{7}, \frac{2}{7}, \frac{2}{7}\right)$ \\
& $\left(\frac{1}{2}, \frac{1}{3}, \frac{3}{7}\right)$ & $\left(\frac{3}{7}, \frac{3}{7}, \frac{3}{7}, \frac{3}{7}, \frac{3}{7}\right)$ \\
\hline 9 & $\left(\frac{1}{2}, \frac{1}{3}, \frac{1}{8}\right)$ & $\left(\frac{1}{2}, \frac{1}{3}, \frac{1}{3}, \frac{1}{3}, \frac{1}{8}\right)$ \\
& $\left(\frac{1}{2}, \frac{1}{3}, \frac{3}{8}\right)$ & $\left(\frac{1}{2}, \frac{1}{3}, \frac{1}{3}, \frac{1}{3}, \frac{3}{8}\right)$ \\
\hline
\end{tabular}

TABLE 2. Exposants de l'équation $\phi_{t}^{*} E$

\section{Solutions algébriques du système de Garnier d'ordre 3}

On s'intéresse maintenant au cas $N=3$ c'est à dire l'application $\phi: \mathbb{P}_{x}^{1} \rightarrow \mathbb{P}_{z}^{1}$ a trois points critiques libres distincts en dehors sur des valeurs critiques $z=0,1$ 
et en $\infty$. Comme la section précédente elle sera définie par une famille de revêtements paramétrés par 3 paramètres. L'équation transformée $E^{\prime}=\phi^{*} E$ par $\phi$ est une équation fuchsienne avec 6 points singuliers non apparents normalisés projectivement à $x=0,1, t_{1}, t_{2}, t_{3}, \infty$ et 3 points singuliers apparents normalisés projectivement à $x=q_{1}, q_{2}, q_{3}$. Les six singularités non apparentes de $E^{\prime}$ sont des points critiques non apparents de $\phi$, toutes sont situées au dessus de l'ensemble $\{0,1, \infty\}$. Tous les points de ramifications de revêtement $\phi$ se trouvent au dessus de $z=0,1, \infty$ sauf 3 points de ramifications simples distincts libres $x=q_{1}, q_{2}, q_{3}$. Alors, d'après la formule de Hurwitz, l'application $\phi$ contient $d+5$ points distincts sur l'ensemble $\{0,1, \infty\}$. Ces trois points critiques libres $x=q_{1}\left(t_{1}, t_{2}, t_{3}\right), x=q_{2}\left(t_{1}, t_{2}, t_{3}\right)$ et $x=q_{3}\left(t_{1}, t_{2}, t_{3}\right)$, des fonctions algébriques en $t_{1}, t_{2}, t_{3}$, sont des solutions algébriques du système de Garnier d'ordre 3. On veut étudier la classification de ces solutions algébriques du système de Garnier de rang 3 provenant du pull-back $\phi$ de l'équation fuchsienne $E$ de monodromies locales $\left(\frac{1}{p_{0}}, \frac{1}{p_{1}}, \frac{1}{p_{\infty}}\right)$. On commence par $E$ équation fuchsienne hypergéométrique, i.e $\frac{1}{p_{0}}+\frac{1}{p_{1}}+\frac{1}{p_{\infty}}<1$ et le groupe de monodromie de l'équation fuchsienne $E^{\prime}$ est irréductible. On a obtenu des résultats similaires à la proposition (3.2) :

Proposition 4.1. - Soit $\phi: \mathbb{P}_{x}^{1} \rightarrow \mathbb{P}_{z}^{1}$ une application revêtement de $\mathbb{P}_{z}^{1}$ de degré $d$, $E$ une équation hypergéométrique fixée sur $\mathbb{P}_{z}^{1}$ avec monodromie $\left(\frac{1}{p_{0}}, \frac{1}{p_{1}}, \frac{1}{p_{\infty}}\right)$ tel que

$$
\frac{1}{p_{0}}+\frac{1}{p_{1}}+\frac{1}{p_{\infty}}<1
$$

Supposons que l'équation fuchsienne obtenue $E^{\prime}=\phi^{*} E$ par le pull-back de l'équation $E$ a 6 points singuliers non apparents normalisés à $0,1, t_{1}, t_{2}, t_{3}$ et $\infty$ d'ordres de monodromies locales autour de chacun de ces points sont respectivement $\tilde{p}_{0}, \tilde{p}_{1}, \tilde{p}_{t_{1}}$, $\tilde{p}_{t_{2}}, \tilde{p}_{t_{3}}$ et $\tilde{p}_{\infty}$ et 3 points singuliers apparents. Alors

1. on a l'égalité $d-\left(\left\lfloor\frac{d}{p_{0}}\right\rfloor+\left\lfloor\frac{d}{p_{1}}\right\rfloor+\left\lfloor\frac{d}{p_{\infty}}\right\rfloor\right)=1$;

2. on obtient l'inégalité $\left(1-\frac{1}{p_{0}}-\frac{1}{p_{1}}-\frac{1}{p_{\infty}}\right) d \leq 1-\frac{6}{p_{\infty}}$;

3. si $p_{\infty}>d$ on $a \frac{1}{d}+\frac{1}{p_{0}}+\frac{1}{p_{1}} \geq 1$;

4. si $p_{\infty} \leq d$ on obtient $\left(1-\frac{1}{p_{0}}-\frac{1}{p_{1}}\right) p_{\infty}^{2}-2 p_{\infty}+6 \leq 0$ et $\frac{5}{6} \leq \frac{1}{p_{0}}+\frac{1}{p_{1}}<1$.

Démonstration. - La démonstration est le même raisonnement que celle de proposition (3.2), mais pour le premier énoncé il faut savoir que le nombre total des points distincts dans les trois fibres de $\phi$ est égal à $d+5$ et pour le deuxième énoncé il faut aussi tenir compte que l'équation fuchsienne $E^{\prime}$ possède 6 points singuliers non apparentes (points singuliers essentiels). Les deux derniers énoncés sont les déductions des deux premiers.

On constate, dans la proposition (4.1), que tous les triplets hyperboliques $\left(p_{0}, p_{1}, p_{\infty}\right)$ ne satisfont pas la condition du quatrième énoncé de proposition. Cela veut dire que $d<p_{\infty}$ alors les points sur $\infty$ sont des points singuliers non apparents de l'équation fuchsienne $E^{\prime}$ et les ordres de monodromie autour de ces points peuvent 
être égaux à l'infini. On obtient la liste des triplets $\left(p_{0}, p_{1}, p_{\infty}\right)$ et ses degrés $d$ satisfaisant la troisième condition :

$-\left(2,3, p_{\infty}\right) d=2,3,4,5,6 ;$

$-\left(2,4, p_{\infty}\right) d=2,3,4$;

$-\left(2, p_{1}, p_{\infty}\right) d=2$;

$-\left(3,3, p_{\infty}\right) d=2,3$.

Si on applique le premier énoncé de la proposition (4.1) certains degrés $d$ de l'application $\phi$ disparaissent. On obtient à la fin la liste des triplets $\left(p_{0}, p_{1}, p_{\infty}\right)$ et de degrés $d$ satisfaisant les énoncés de la proposition (4.1) :

\begin{tabular}{|c|c|}
\hline Triplets $\left(p_{0}, p_{1}, p_{\infty}\right)$ & Degrés $d$ \\
\hline$\left(2,3, p_{\infty}\right)$ & $2,3,4,6$ \\
\hline$\left(2,4, p_{\infty}\right)$ & 2,4 \\
\hline$\left(2, p_{1}, p_{\infty}\right)$ & 2 \\
\hline$\left(3,3, p_{\infty}\right)$ & 3 \\
\hline
\end{tabular}

TABLE 3. Triplets et Revêtements correspondants

On cherche maintenant des revêtements qui transforment l'équation hypergéométrique $E$ à l'équation fuchsienne avec $6+3$ pôles simples. Ils seront choisi parmi les éléments préliminairement selectionnés dans le tableau (3), on a eu le résultat suivant.

Théorème 4.2. - Les solutions algébriques du système de Garnier d'ordre 3 avec groupe de monodromie irréductible infini (Zariski dense) sont construites par la déformation isomonodromique de l'équation fuchsienne obtenue $E^{\prime}$ par le pull-back $\phi$ de l'équation hypergéométrique E. Alors il existe un et un seul revêtement $\phi$ à homographie près de degré d et de type de ramification listé dans le tableau:

\begin{tabular}{|c|c|c|c|}
\hline \multicolumn{2}{|c|}{ Monodromies } & Degré de $\phi$ & $\begin{array}{c}\text { Type de revêtement } \\
(\cdots ; \cdots ; \cdots)\end{array}$ \\
\cline { 1 - 2 } Équation $E$ & Équation $E^{\prime}$ & $d$ & $(2+2+2 ; 3+3 ; \underbrace{1+\cdots+1}_{\text {6fois }})$ \\
\hline
\end{tabular}

TABlE 4. Transformation de l'équation hypergéométrique à l'équation fuchsienne avec $6+3$ singuliers.

Démonstration. - D'après la proposition (4.1) pour tous les triplets $\left(p_{0}, p_{1}, p_{\infty}\right)$ fixés sur $\mathbb{P}_{z}^{1}$, on a $d<p_{\infty}$. On a l'inégalité $\frac{d-s_{0}}{p_{0}}+\frac{d-s_{1}}{p_{1}}+\frac{d-s_{\infty}}{p_{\infty}} \geq d-1$ due à la formule de Riemann-Hurwitz [16] où $s_{0}, s_{1}$ et $s_{\infty}$ sont le nombre de points critiques non apparents sur $i=0,1$ et $\infty$ respectivement telle que $\sum_{i=0,1, \infty} s_{i} \geq 6$. Si on fixe l'équation 
fuchsienne sur $\mathbb{P}_{z}^{1}$ avec triplet $\left(2,3, p_{\infty}\right)$. Dans le tableau (3) le degré maximal correspondant à ce triplet est 6 et on peut supposer que $p_{\infty} \geq 7$ alors l'inégalité ci-dessus devient

$$
2 \leq d \leq 6-15 s_{0}-8 s_{1} .
$$

On a une seule possibilité sur $s_{0}$ et sur $s_{1}$ pour trouver une solution de l'inégalité

$$
s_{0}=s_{1}=0 \text { et } s_{\infty} \geq 6 \Rightarrow d \leq 6 .
$$

Cela veut dire que tous les points critiques non-apparents sont sur $\infty$ et les points sur 0 et sur 1 sont tous critiques apparents alors le degré $d$ doit être multiple commun de 2 et 3 . On a une seule valeur de $d \in[2,6]$, que 2 et 3 lui divisent, est $d=6$. On a une seule façon de partition de $d$ au dessus de 0 et de 1 pour trouver le nombre maximal de points critiques apparents. On a sur 0 trois points critiques apparents et sur 1 deux points critiques apparents. Donc on obtient le type de ramification de $\phi$ est donné par $(2+2+2 ; 3+3 ; 1+1+1+1+1+1)$.

Si on considère l'équation fuchsienne $E$ correspondante au triplet $\left(2,4, p_{\infty}\right)$ avec $p_{\infty} \geq$ 5 ; puisque dans le tableau (3) le degré maximal correspondant au triplet $\left(2,4, p_{\infty}\right)$ est $d=4$; on a l'inégalité

$$
d \leq-4-6 s_{0}-s_{1}
$$

Alors $d$ est majoré par un nombre entier négatif contredit du fait que $2 \leq d<$ $p_{\infty}$. Donc il n'existe pas de revêtement $\phi$ qui tire en arrière l'équation fuchsienne hypergéométrique $E$, avec paramètre $\left(\frac{1}{2}, \frac{1}{4}, \frac{1}{p_{\infty}}\right)$, à l'équation fuchsienne $E^{\prime}$.

Pour le triplet $(2, p, p)$, il est associé à une seule application de degré 2 . Alors quel que soit la répartition de 2 , le nombre des points distincts dans les trois fibres ne vaut pas 7 .

Pour $\left(3,3, p_{\infty}\right)$ où $p_{\infty} \geq 4$; pour toutes les valeurs de $s_{0}$ et $s_{1}$ on a $d \leq-6-s_{0}-s_{1}$ cela contredit aussi $2 \leq d<p_{\infty}$.

\section{Système de Garnier d'ordre supérieur ou égal 4}

On suppose que l'équation fuchsienne obtenue $E^{\prime}$ après la transformation de $E$ par $\phi$ possède $n \geq 7$ points singuliers non apparents et $N \geq 4$ singularités apparentes. Si on suppose de plus que l'équation fuchsienne $E$ est hypergéométrique, le deuxième et le quatrième énoncés de la proposition (4.1) modifient respectivement en

1. $\left(1-\sum_{i=0,1, \infty} \frac{1}{p_{i}}\right) d \leq 1-\frac{n}{p_{\infty}}$,

2. $p_{\infty} \leq d$ on a $\left(1-\frac{1}{p_{0}}-\frac{1}{p_{1}}\right) p_{\infty}^{2}-2 p_{\infty}+n \leq 0$ et $\frac{n-1}{n} \leq \frac{1}{p_{0}}+\frac{1}{p_{1}}<1$.

On remarque dans la section précédente qu'à partir de $n=6$ le degré de revêtements existant est strictement inférieur à $p_{\infty}$. Les éléments listés dans le tableau (3) restent les mêmes, aucun revêtement ne peut transformer l'équation $E$ en équation fuchsienne $E^{\prime}$. 
Théorème 5.1. - Si $n \geq 7$ il n'existe pas une solution algébrique complète du système de Garnier, avec groupe de monodromies irréductibles, obtenue par la méthode de Kitaev.

Démonstration. - D'après la formule de Riemann-Hurwitz, on a $\frac{d-s_{0}}{p_{0}}+\frac{d-s_{1}}{p_{1}}+$ $\frac{d-s_{\infty}}{p_{\infty}} \geq d-1$, où $s_{0}, s_{1}$ et $s_{\infty}$ sont respectivement le nombre de points critiques non apparents sur 0,1 et $\infty$ tel que $\sum_{i=0,1, \infty} s_{i}=n$. Si on fixe le triplet $(2,3, p)$, on obtient l'inégalité ci-dessus sous la forme

$$
d \leq 6(7-n)-15 s_{0}-8 s_{1} .
$$

Si $3 \leq n \leq 6$ on obtient des solutions algébriques aux déformations de deux équations fuchsiennes déjà connues et aux deux autres équations que nous avons fait en haut. Si $n \geq 7$, le degré $d$ du revêtement $\phi$ dans l'inégalité (6) est majorée par des entiers naturels négatifs, alors $\phi$ n'existe pas. Cela entraîne la non existence de l'équation fuchsienne $E^{\prime}$.

On voit qu'on ne peut plus trouver un revêtement $\phi$ dès que $n>6$ et on peut tester les autres types :

triplet $(2,4, p)$ : on a l'inégalité $d \leq 4(5-n)-6 s_{0}-s_{1}$ si $n \geq 6$;

le triplet $(3,3, p)$ : on obtient $d \leq 3(4-n)-s_{0}-s_{1}$ si $n \geq 5$.

\section{Exemple : Revêtement degré 4}

On veut calculer explicitement le revêtement $\phi: \mathbb{P}_{x}^{1} \rightarrow \mathbb{P}_{z}^{1}$ de degré 4 du tableau (11). À homographie près en $z$, le type de ramification recherché est $(2+2 ; 1+1+1+1 ; 3+1)$ :

- la fibre $\phi^{-1}(0)$ est totalement ramifiée à l'ordre 2 ,

- la fibre $\phi^{-1}(1)$ est non ramifiée, constituée des points $x=0,1, t_{1}, t_{2}$,

- la fibre $\phi^{-1}(\infty)$ a un point simple $x=\infty$ et un point triple,

- $\phi$ a deux autres points critiques $x=q_{1}, q_{2}$.

Ces contraintes nous imposent que l'application $\phi$ est de la forme

$$
\phi(x)=-\frac{c^{3}}{a_{0}^{2}} \frac{\left(x^{2}+a_{1} x+a_{0}\right)^{2}}{(x-c)^{3}}
$$

où les constantes $a_{0}, a_{1}, c$ satisfont $(\phi(1)=1)$

$$
\frac{a_{0}^{2}}{c^{3}}+\frac{\left(1+a_{1}+a_{0}\right)^{2}}{(1-c)^{3}}
$$

Cette équation définit une surface rationnelle $S$ que l'on peut paramétrer par $\left(a_{0}, s\right)$ :

$$
a_{1}=a_{0}\left(s^{3}-1\right)-1 \quad \text { et } \quad c=\frac{1}{1-s^{2}} .
$$

Les deux points $x=t_{1}, t_{2}$ sont alors les racines de l'équation polynômiale

$$
t^{2}-\left(a_{0}^{2}\left(s^{2}-1\right)^{3}-2 a_{0}\left(s^{3}-1\right)+1\right) t+a_{0}\left(2-a_{0}\left(2 s^{3}-3 s^{2}+1\right)\right)=0
$$


qui définit une nouvelle surface $S_{t}$ : la projection $S_{t} \rightarrow S ;\left(t, a_{0}, s\right) \mapsto\left(a_{0}, s\right)$ est un revêtement double ramifiant le long du discriminant du polynôme précédent. C'est encore une surface rationnelle que l'on peut paramétrer par $(s, t)$ en posant

$$
a_{0}=\frac{t^{2}-1}{(s+1)\left((s-1)^{2} t^{2}-(s+1)^{2}\right)} .
$$

On obtient alors les solutions $x=t_{1}, t_{2}$ :

$$
t_{1}=\frac{-(t+1)(s t-t-1-3 s)}{(s t-t-1-s)^{2}(s+1)} \quad \text { et } \quad t_{2}=-\frac{(-1+t)(s t-t+1+3 s)}{(s t-t+s+1)^{2}(s+1)} .
$$

On vérifie sans peine que l'application

$$
\Pi_{t}: S_{t} \rightarrow \mathbb{P}^{1} \times \mathbb{P}^{1} ;(s, t) \mapsto\left(t_{1}, t_{2}\right)
$$

est de degré 8 , ramifiant précisément au dessus de

$$
t_{1}=0,1, \infty, \quad t_{2}=0,1, \infty \quad \text { et la diagonale } \quad t_{1}=t_{2} .
$$

C'est la propriété de Painlevé. Il nous reste à déterminer les deux points critiques libres $x=q_{1}, q_{2}$. Ils sont solutions de l'équation polynômiale

$$
\begin{aligned}
& x^{2}-\frac{t^{2} s^{3}+3 s^{3}-4 t^{2} s^{2}+4 s^{2}+5 t^{2} s+7 s+2-2 t^{2}}{(s-1)(s+1)(s t-t+s+1)(s t-t-1-s)} x \\
& -\frac{(s t-t+1+3 s)(s t-t-1-3 s)}{(s+1)^{2}(s t-t+s+1)(s t-t-1-s)(s-1)}=0
\end{aligned}
$$

dont le discriminant est donné par $s^{2}(s+1)^{2} F(s, t)$ avec

$$
\begin{gathered}
F(s, t)=s^{4} t^{4}+6 s^{4} t^{2}+9 s^{4}-4 t^{4} s^{3}-56 t^{2} s^{3}+60 s^{3}+6 t^{4} s^{2}+100 t^{2} s^{2} \\
+118 s^{2}-4 t^{4} s-56 t^{2} s+60 s+t^{4}+6 t^{2}+9 .
\end{gathered}
$$

Pour déterminer les racines $x=q_{1}, q_{2}$, il nous faut passer de nouveau à un revêtement double $S_{q} \rightarrow S_{t}$ ramifiant le long de la courbe algébrique $F(s, t)=0$ (afin que le discriminant devienne un carré). Cette courbe est irréductible sur $\mathbb{Q}$ mais réductible sur $\mathbb{Q}(\alpha)$, avec $\alpha^{2}+3=0$. On obtient que la courbe $F(s, t)=0$ est la réunion de deux coniques $F_{1}$ et $F_{2}$ qui s'intersectent en quatre points où

$$
\begin{aligned}
& F_{1}(s, t)=t^{2}+2 t \alpha-3-2 t^{2} s-4 s t \alpha-10 s+t^{2} s^{2}+2 s^{2} t \alpha-3 s^{2} \\
& F_{2}(s, t)=t^{2} s^{2}-2 s^{2} t \alpha-3 s^{2}-2 t^{2} s+4 s t \alpha-10 s+t^{2}-2 t \alpha-3 .
\end{aligned}
$$

En paramétrant le pinceau de coniques associés, on obtient une paramétrisation rationnelle $(u, v)$ pour $S_{q}$ en posant

$$
s=-\frac{4 u\left(v^{\prime} u+2\right)}{u^{2}-3-u v^{\prime}}, \quad t=-\frac{3 u^{2}+v^{\prime} u-1}{u^{2}-3-v^{\prime} u} \quad \text { avec } \quad \frac{-2 \alpha+v^{\prime}}{2 \alpha+v^{\prime}}=v^{2} .
$$


On obtient enfin la paramétrisation complète de la solution algébrique (rationnelle $\operatorname{sur} \mathbb{Q}(\alpha))$ :

$$
\begin{aligned}
t_{1}(u, v)= & -\frac{1}{52}(353+9 \alpha)\left(-4 \alpha u^{2}+2 u v^{2}-2 u-v^{2}+1+13 u^{2} v^{2}+11 u^{2}+4 \alpha u v^{2}\right. \\
& \left.-4 \alpha u-2 \alpha v^{2}+2 \alpha\right) u(2 v-1+\alpha)(2 v+1-\alpha)(u v+u+\alpha v-\alpha)^{2}(u v-u \\
& +\alpha v+\alpha)^{2} /\left((u+1)\left(u^{2} v^{2}-u^{2}-2 \alpha u v^{2}-2 \alpha u+v^{2}-1\right)(v+1)(v-1)(\alpha v\right. \\
& \left.-2 v+\alpha-2+7 u v+u-4 \alpha u)^{2}(\alpha v-2 v+2-\alpha+7 u v-u+4 \alpha u)^{2}\right) ; \\
t_{2}(u, v)= & \frac{1}{52}(9 \alpha-353)\left(-v^{2}+1+13 u^{2} v^{2}+11 u^{2}+4 \alpha u^{2}-2 u v^{2}+2 u+2 \alpha v^{2}\right. \\
& \left.-2 \alpha+4 \alpha u v^{2}-4 \alpha u\right) u(2 v-1-\alpha)(2 v+1+\alpha)(u v+u+\alpha v-\alpha)^{2}(u v \\
& -u+\alpha v+\alpha)^{2} /\left((u-1)\left(u^{2} v^{2}-u^{2}-2 \alpha u v^{2}-2 \alpha u+v^{2}-1\right)(v+1)(v\right. \\
& -1)(2 v+\alpha v-2-\alpha+7 u v-u-4 \alpha u)^{2}(2 v+\alpha v+2+\alpha \\
& \left.+7 u v+u+4 \alpha u)^{2}\right) ; \\
q_{1}(u, v)= & -\frac{7}{2}(u v-u+\alpha v+\alpha) u(2 v+1-\alpha)(2 v+1+\alpha)(u v+u+\alpha v \\
& -\alpha)^{2} /\left(\left(u^{2} v^{2}-u^{2}-2 \alpha u v^{2}-2 \alpha u+v^{2}-1\right)(\alpha v-2 v+2-\alpha+7 u v-u\right. \\
& +4 \alpha u)(2 v+\alpha v-2-\alpha+7 u v-u-4 \alpha u)(v+1)) ; \\
& -\frac{7}{2}(u v+u+\alpha v-\alpha) u(2 v-1+\alpha)(2 v-1-\alpha)(u v-u+\alpha v \\
& +\alpha)^{2} /\left(\left(u^{2} v^{2}-u^{2}-2 \alpha u v^{2}-2 \alpha u+v^{2}-1\right)(2 v+\alpha v+2+\alpha+7 u v+u\right. \\
& +4 \alpha u)(\alpha v-2 v+\alpha-2+7 u v+u-4 \alpha u)(v-1)) .
\end{aligned}
$$

\section{Références}

[1] J. M. Couveignes, Calcul et rationalité de fonctions de Belyi, Annales de l'institut Fourier, tome $44, \mathrm{n}^{\circ} 1$ (1994), p. $1-38$.

[2] G. V. Belyi, Galois extensions of a maximal cyclotomic field. (Russian), Izv. Akad. Nauk SSSR Ser. Mat. 43 (1979), no. 2, 267-276, English translation in Math. USSR Izv. 14 (1980), 247-256.

[3] P. Boalch, From Klein to Painlevé via Fourier, Laplace and Jimbo, Proc. London Math. Soc. (3) 90(2005), p. $167-208$.

[4] P. Boalch, The fifty-two icosahedral solutions to Painlevé VI, J. Reine Angew. Math. 596 (2006), p. $183-214$.

[5] P. Boalch, Some explicit solutions to the Riemann-Hilbert problem, IRMA Lectures in Mathematics and Theoretical Physics, vol. 9 (2006) p. $85-112$.

[6] C. F. Doran, Algebraic and Geometric Isomonodromic Deformations, J. Differential Geometry 59 (2001), 33-85.

[7] B. Dubrovin and M. Mazzocco, Monodromy of certain Painlevé VI Transcendents and Reflection Groups, Ivent. Math. 141 (2000), p. 55 - 147. 
[8] B. Dubrovin and M. Mazzocco, Canonical structure and symmetries of the Schlesinger equations, Comm. Math. Phys. 271(2007),nº . 2, p. $289-373$.

[9] R. Garnier, Sur des systèmes différentiels du second ordre dont l'intégrale générale est uniforme, Ann. Sci. École Norm. Sup. $3^{\text {e }}$ série, tome 77, no 2 (1960), p. 123-144.

[10] N. J. Hitchin, Poncelet Polygons and the Painlevé Equations, Geometry and analysis (Bombay, 1992), Tata Inst. Fund. Res., Bombay, 1995, p.151 - 185.

[11] N. J. Hitchin, Twistor spaces, Einstein metrics and isomonodromic deformations, J. Differential Geom. 42(1995), nº . 1, p. $30-112$.

[12] Mr. J. Hodgkinson, A detail in Conformal Representation, Proc. London Math. Soc., Ser. 2, Vol. 15(1916), p. $166-181$.

[13] K. Iwasaki, H. Kimura, S. Shimomura, S. Yoshida, From Gauss to Painlevé : A Modern Theory of Special Functions, Braunschweig : Vieweg, (1991), 347pages.

[14] Jimbo and T. Miwa, Monodromy preserving deformation of linear ordinary differential equations with rational coefficients II, Physica $2 D$ (1981), p.407 - 448.

[15] A. V. Kitaev and R. Vidanus, Transformations $R S_{4}^{2}(3)$ of the Ranks $\leq 4$ and Algebraic Solutions of the Sixth Painlevé Equation, Comm. Math. Phys. 228 (2002), p.151 - 176.

[16] A. Kitaev, Grothendieck's Dessin d'Enfants, Their Deformations and Algebraic Solutions of the Sixth Painlevé and Gauss Hypergeometric Equations, Algebra $i$ Analiz 17, $\mathrm{n}^{\circ}$. 1 (2005), p. $224-273$.

[17] A. V. Kitaev, Remarks Towards the Classification of $R S_{4}^{2}(3)$-Transformations and Algebraic Solutions of the Sixth Painlevé Equation, Sémin. Congr., 14, Soc. Math. France, Paris, (2006), p. $199-227$.

[18] F. Klein, Vorlesungen über das Ikosaedar, B. G. Teubner, Leipzig, (1884).

[19] O. Lisovyy, Y. Tykhyy, Algebraic Solutions of the sixth Painlevé Equation, Preprint http ://arxiv.org/abs/0809.4873v2 (2008)

[20] H. P. de Saint-Gervais, Uniformisation des surfaces de Riemann. Retour sur un théorème centenaire, ENS Éditions École normale supérieure de Lyon (2011) 544 pages.

[21] H. Watanabe, Birational canonical transformations and classical solutions of the sixth Painlevé equation, Ann. Scuola Norm. Sup. Pisa Cl. Sci. 27, (1999), p.379-425.

K. DIARRA, IRMAR, Unité Mixte de Recherche 6625 du CNRS, Université de Rennes 1, Campus de Beaulieu, 35042 Rennes Cedex, France, DER de Mathématiques et d'informatique, FAST, Université de Bamako, BP : E 3206 Mali. • E-mail : diarak2005@yahoo.fr E-mail : karamoko.diarra@univ-rennes1.fr 